Research, Society and Development, v. 9, n. 11, e2339119006, 2020

(CC BY 4.0) | ISSN 2525-3409 | DOI: http://dx.doi.org/10.33448/rsd-v9i11.9006

\title{
Realização do exame citopatológico em mulheres: uma revisão integrativa
}

Cytopathological examination in women: an integrative review

Examen citopatológico en mujeres: una revisión integradora

Recebido: 03/10/2020 | Revisado: 12/10/2020 | Aceito: 08/11/2020 | Publicado: 12/11/2020

Giovanna Stefanne Lópes Barbosa

ORCID: https://orcid.org/0000-0003-3387-1219

Universidade Federal do Delta do Parnaíba, Brasil.

E-mail: gistefanne@gmail.com

Antônio Tiago da Silva Souza

ORCID: https://orcid.org/0000-0003-1904-1681

Universidade Federal do Delta do Parnaíba, Brasil

E-mail: at.tiago@hotmail.com

Francisco Clezion Franca Vasconcelos Júnior

ORCID: https://orcid.org/0000-0002-8460-3903

Universidade Federal do Ceará, Brasil

E-mail: clezionjr@gmail.com

Edmar José Fortes Júnior

ORCID: https://orcid.org/0000-0002-0114-9937

Instituto de Educação Superior do Vale do Parnaíba, Brasil.

E-mail: edmarfortes@ hotmail.com

Daniel Mayco de Melo Oliveira

ORCID: https://orcid.org/0000-0003-1137-6209

Faculdade Metropolitana de Manaus, Brasil

E-mail: danielmaicooliveira@hotmail.com

Francisco Lukas Rodrigues Martins

ORCID: https://orcid.org/0000-0003-4454-5849

Universidade Federal do Delta do Parnaíba, Brasil

E-mail: lukasmartins_play88@hotmail.com

Francisco Ricardo Nascimento Freitas

ORCID: https://orcid.org/0000-0003-2655-619X

Universidade Federal do Delta do Parnaíba, Brasil

E-mail: ricardofreitasac@gmail.com 


\section{Hyan Crysthyan Apolinário Silveira}

ORCID: https://orcid.org/0000-0003-2900-3451

Universidade Federal do Delta do Parnaíba, Brasil

E-mail: hyan0800@hotmail.com

Ivy Louise Barros

ORCID: https://orcid.org/0000-0003-3622-1078 Universidade Federal do Delta do Parnaíba, Brasil

E-mail: ivybarros14@ hotmail.com

Leonardo Vitor Nunes de Oliveira

ORCID: https://orcid.org/0000-0002-2579-7509

Universidade Federal do Delta do Parnaíba, Brasil

E-mail: leonardo_oliveira28@hotmail.com

Mariana Veras Rocha Borges

ORCID: https://orcid.org/0000-0003-2922-4377

Universidade Federal do Delta do Parnaíba, Brasil

E-mail: mariana_vrborges@ @otmail.com

Nayanna de Almeida Nassif Rodrigues

ORCID: https://orcid.org/0000-0002-2883-5398

Universidade Federal Fluminense, Brasil

E-mail: nayannanassif@ outlook.com

Rafaela Sartori Tonin

ORCID: https://orcid.org/0000-0002-7529-634X

Pontifícia Universidade Católica do Paraná, Brasil

E-mail: rafasartoritonin@hotmail.com

Raimundo Graças Almeida Lima Neto

ORCID: https://orcid.org/0000-0001-6454-3838

Universidade Federal do Delta do Parnaíba, Brasil

E-mail: neto.poseidon7@gmail.com

Samuel Aragão Cansanção Bona Ibiapina

ORCID: https://orcid.org/0000-0002-9521-214X

Instituto de Educação Superior do Vale do Parnaíba, Brasil

E-mail: samuel_ibiapina@hotmail.com 


\section{Carlos Alberto Teixeira Costa}

ORCID: https://orcid.org/0000-0002-6498-8682

Universidade Federal do Delta do Parnaíba, Brasil

E-mail: carlosteixeira@globo.com

Daniela França de Barros

ORCID: https://orcid.org/0000-0002-3667-7510

Universidade Federal do Delta do Parnaíba, Brasil

E-mail: danifbarros@bol.com.br

José Ivo dos Santos Pedrosa

ORCID: https://orcid.org/0000-0002-5416-2860

Universidade Federal do Delta do Parnaíba, Brasil

E-mail: jivopedrosa@gmail.com

\section{Resumo}

Objetivou-se levantar na literatura os principais fatores de adesão e não adesão ao exame Papanicolau, principal meio de prevenção do Câncer de Colo do Útero (CCU). Realizou-se revisão de literatura nos bancos de dados BVS, BDNEF, LILASC e SciELO, entre 2013 e 2020, utilizando os descritores: teste de Papanicolau e câncer do colo do útero. Elaboraram-se três categorias: principais fatores de adesão e não adesão à realização do exame Papanicolau; fatores de risco para o CCU e caracterização sociodemográfica. Os resultados deste estudo revelaram diversos fatores que influenciam a mulher a realizar o exame preventivo do $\mathrm{CCU}$, e em número mais significativo os fatores que levam as mulheres a não aderir ao exame. Percebeu-se que as mulheres com grau de escolaridade mais avançado, que conheciam a importância do exame, que possuíam entendimento do CCU e uma melhor renda familiar, o exame citopatológico estava presente na sua rotina de cuidado com a saúde. Vale salientar que há uma limitação da realização do exame citopatológico nas mulheres de baixa renda, com baixo nível de escolaridade, conhecimento insuficiente sobre o exame e patologia e, principalmente, as que possuem o sentimento de vergonha em relação à realização do exame.

Palavras-chave: Teste de Papanicolau; Neoplasias do colo do útero; Programas de rastreamento. 


\section{Abstract}

The main objective of this study is to raise in the literature the main factors of adherence and non-adherence to the Pap smear test as the main means of preventing Cervical Cancer (CC). A literature review was carried out in the VHL, BDNEF, LILASC and SciELO databases, between 2013 and 2020, using the descriptors: Pap smear test and cervical cancer. Three categories were elaborated: main factors of adherence and non-adherence to the Pap smear test; risk factors for $\mathrm{CC}$ and sociodemographic characterization. The results of this study revealed several factors that influence women to undergo the $\mathrm{CC}$ preventive exam, and in a more significant number the factors that lead women to not adhere to the exam. It was noticed that women with more advanced schooling, who knew the importance of the exam, who had an understanding of cervical cancer and a better family income, the cytopathological exam was present in their health care routine. It is worth mentioning that there is a limitation in the performance of the cytopathological examination in low-income women, with low level of education, insufficient knowledge about the examination and pathology and especially those who have a feeling of shame in relation to the examination.

Keywords: Papanicolaou test; Uterine cervical neoplasms; Mass screening.

\section{Resumen}

El objetivo principal de este estudio es plantear en la literatura los principales factores de adherencia y no adherencia a la prueba de Papanicolaou como principal medio de prevención del Cáncer Cervical (CC). Se realizó una revisión de la literatura en las bases de datos VHL, BDNEF, LILASC y SciELO, entre 2013 y 2020, utilizando los descriptores: prueba de Papanicolaou y cáncer de cuello uterino. Se elaboraron tres categorías: principales factores de adherencia y no adherencia a la prueba de Papanicolaou; factores de riesgo para CC y caracterización sociodemográfica. Los resultados de este estudio revelaron varios factores que influyen en las mujeres para someterse al examen preventivo $\mathrm{CC}$, y en un número más significativo los factores que llevan a las mujeres a no adherirse al examen. Se notó que las mujeres con escolaridad más avanzada, que conocían la importancia del examen, que tenían un conocimiento del cáncer de cuello uterino y un mejor ingreso familiar, el examen citopatológico estaba presente en su rutina asistencial. Cabe mencionar que existe una limitación en la realización del examen citopatológico en mujeres de bajos ingresos, con bajo nivel educativo, conocimiento insuficiente sobre el examen y patología y especialmente aquellas que tienen un sentimiento de vergüenza en relación al examen.

Palabras-clave: Prueba de Papanicolaou; Neoplasias del cuello uterino; Tamizaje masivo. 


\section{Introdução}

O câncer do colo do útero é o quarto tipo mais frequente entre as neoplasias malignas do sexo feminino, com aproximadamente 530 mil novos casos por ano no mundo. Esta é responsável, pelo óbito de 265 mil mulheres anualmente, apresentando-se a quarta causa de morte por câncer entre elas. No Brasil, em 2020, foram estimados 16.710 casos novos (INCA, 2019). Dados do Sistema de Informações de Mortalidade contabilizam 6.526 óbitos por neoplasia maligna do colo do útero em 2018 (Brasil, 2020).

O câncer do colo do útero é uma patologia que se desenvolve lentamente, podendo não manifestar sinais e sintomas em fase inicial. Pode ser causado pelo Papiloma Vírus Humano (HPV), que tem como principal forma de transmissão à relação sexual. Nas mulheres jovens, a infecção por HPV pode ser transitória, com regressão espontânea, sem que exista necessidade de tratamento (Rodrigues, Moreira, Alves, \& Guimarães, 2016).

Dentre os fatores que podem influenciar no aparecimento do câncer do colo uterino, vale destacar: tabagismo, vida sexual precoce, múltiplos parceiros sexuais, uso de contraceptivos orais, multiparidade, baixa condição socioeconômica, entre outros. Um importante fator de risco para o surgimento dessa doença é a infecção pelo HPV, microrganismo associado à maioria dos casos de lesão precursora deste tipo de câncer. Essa lesão pode ser detectada precocemente, a partir da realização do exame citopatológico (Souza \& Costa, 2015).

Estes fatores, quando manejados da forma adequada, podem desencadear um diagnóstico precoce da doença. Quando esse diagnóstico não ocorre e o câncer do colo do útero tem descoberta tardia, as taxas de mortalidade na população brasileira tornam-se elevadas. Pontua-se que o seu diagnóstico deva ocorrer em tempo hábil para um tratamento adequado, assim, aumentando à possibilidade de cura.

No Brasil, o rastreamento do câncer do colo do útero é desenvolvido por meio do exame citopatológico, conhecido como teste de Papanicolau, um método simples e de baixo custo, que permite identificar alterações no epitélio cervical que indiquem a presença de lesões precursoras deste tipo de câncer ou a própria patologia (Ribeiro \& Andrade, 2016).

O Ministério da Saúde preconiza que o rastreamento do câncer do colo uterino seja realizado em mulheres com faixa etária de 25 a 64 anos. Nas mulheres a partir de 25 anos e que tenham vida sexual ativa, quando é realizado anualmente e com dois resultados negativos, o intervalo deve ser de três anos entre os exames. Essa recomendação segue para todas as mulheres até os 64 anos, quando somente deve ser interrompido quando há pelo menos dois exames negativos nos últimos cinco anos (Nascimento, Pereira, Nascimento, Lourenço, \& 
Machado, 2015).

Sem dúvidas, o exame citopatológico é considerado efetivo e eficaz. Sendo que as mulheres que realizam o exame têm uma maior possibilidade de diagnóstico precoce, redução do número de indivíduos acometidos em fases avançadas da doença, aumentando as chances de tratamento. Mas sua cobertura ainda é insuficiente, devido a diferentes fatores, como sentimentos, crenças, atitudes, aspectos socioeconômicos e a acessibilidade (Carvalho et al., 2016).

Após colocações até aqui traçadas e discutidas, ressalta-se que o exame citopatológico se torna uma das ferramentas fundamentais para a redução das taxas e índices epidemiológicos desfavoráveis relacionados com o câncer de colo do útero. Por conseguinte, este exame assume um papel determinante para as mulheres que se encontram na faixa etária de 25 a 64 anos. Da mesma forma, o acompanhamento destas pelo profissional de saúde e a realização periódica desse exame demonstram que elas estão aderindo às medidas de cuidados preventivos com sua saúde.

A detecção precoce do câncer de colo do útero tem um papel relevante para a adoção do tratamento adequado e em consequência a cura e ou controle da neoplasia abordada. Na maioria dos casos, existe uma maior probabilidade de eficácia com a incorporação do exame Papanicolau pelas mulheres alvo desta ação.

Para colaborar com essa discussão, o presente estudo teve como objetivo levantar na literatura os principais fatores de adesão e não adesão ao exame Papanicolau como principal meio de prevenção do câncer do colo de útero, por meio de revisão de literatura.

\section{Metodologia}

Souza, Silva e Carvalho (2010), delineiam revisão integrativa como sendo um método que proporciona a sinopse de conhecimento, permitindo a aplicação dos resultados na prática, constituindo um instrumento de prática baseada em evidências. Este tipo de estudo, baseia-se em uma abordagem metodológica que permite a inclusão de estudos experimentais e não experimentais. Entretanto, com base no estudo realizado, foi desenvolvida uma revisão integrativa abordando a realização do exame citopatológico por mulheres.

A revisão integrativa tem na sua composição diferentes fases, sendo elas: identificação do tema ou questionamento, busca na literatura, categorização dos estudos, avaliação dos estudos que foram incluídos na pesquisa, interpretação dos resultados e a síntese do 
conhecimento evidenciado pela análise dos artigos selecionados (Pompeo, Rossi, \& Galvão, 2009).

Segundo Botelho, Cunha e Macedo (2011), a etapa subsequente à escolha do tema que será abordado no estudo é a elaboração da pergunta norteadora, sendo que a mesma deve ser formulada de forma compreensível e objetiva, possibilitando ao leitor a ideia central da pesquisa. Desse modo, o estudo apresenta a seguinte pergunta norteadora: Qual a prevalência da realização do exame citopatológico por mulheres?

Buscaram-se artigos para a realização do presente estudo no banco de dados da Biblioteca Virtual em Saúde (BVS), utilizando os artigos da Base de Dados de Enfermagem (BDENF) e Literatura Latino-Americana e do Caribe em Ciências da Saúde (LILACS), como também da base de dados da Scientific Electronic Library Online (SciELO). Essas bases de dados online foram escolhidas por serem mais conhecidas em território nacional, e por apresentarem um grande número de periódicos indexados a elas.

Como critérios de inclusão, foram utilizados artigos publicados no período de 2013 a 2020, cujo tema estivesse relacionado diretamente ao tema do presente estudo; artigos com resumo e texto completo disponível; artigos publicados em língua portuguesa; artigos que abordavam a realização do exame citopatológico, e que apresentavam o câncer do colo do útero como foco do estudo.

Dentre os critérios de exclusão, estão os artigos cujo tema não abordava as temáticas "câncer do colo do útero" e "realização do exame citopatológico"; não tinham o texto completo disponível para consulta; artigos que abordavam o tema com informações vagas ou repetidas; artigos que não estavam em língua portuguesa; artigos publicados em um período anterior ao ano de 2013; artigos que não respondiam ao longo de sua abordagem o questionamento proposto ao estudo; e, por fim, também se considerou a ocorrência de duplicidade nas diferentes bases de dados, sendo que na ocorrência desta, contabilizou-se o estudo apenas em uma base de dados.

Inicialmente utilizaram-se como Descritores em Ciências da Saúde: “Teste de Papanicolau" e "câncer de colo do útero". Realizando-se a busca com a integração dos mesmos em pares por meio da utilização de operadores booleanos (AND, AND OR e NOT).

Após a busca dos artigos, respeitando os critérios de inclusão e exclusão, foram selecionados 15 artigos. Os dados dos artigos selecionados foram agrupados em uma tabela que sintetizou todas as informações relevantes à discussão dos resultados do estudo. O instrumento utilizado destacou as informações referentes ao autor, banco de dados, ano de publicação, local, objetivos, tipo de estudo, amostra e principais achados. 


\section{Resultados e Discussão}

Os artigos selecionados para esta revisão integrativa encontram-se listados na Tabela 1, detalhados quanto à autoria, ano de publicação, base de dados onde foi identificado, local do estudo, objetivos, tipo de estudo, amostra e principais achados. Em seguida é realizada a discussão de literatura.

Tabela 1. Disposição dos estudos utilizados na revisão. Parnaíba, 2020.

\begin{tabular}{|c|c|c|c|}
\hline $\begin{array}{c}\text { Autores (Ano de } \\
\text { publicação)/ Base de } \\
\text { dados }\end{array}$ & Local & Objetivos & $\begin{array}{c}\text { Tipo de } \\
\text { estudo/Amostra/Principais } \\
\text { achados } \\
\end{array}$ \\
\hline $\begin{array}{l}\text { Ceolin, Nasi, Coelho, } \\
\text { Paz e Lacchini. } \\
\text { (2020)/LILACS }\end{array}$ & $\begin{array}{l}\text { Três Passos } \\
\text { (RS) }\end{array}$ & $\begin{array}{c}\text { Analisar o } \\
\text { rastreamento do } \\
\text { câncer do colo do } \\
\text { útero de um } \\
\text { município do Sul } \\
\text { do Brasil. }\end{array}$ & $\begin{array}{l}\text { Estudo quantitativo } \\
\text { descritivo retrospectivo } \\
\qquad \text { (n=1.583) } \\
\text { Foi observado que a maioria } \\
\text { dos exames (80,3\%) foi } \\
\text { realizado pelas mulheres } \\
\text { alvo do programa de } \\
\text { rastreamento de câncer de } \\
\text { colo de útero (entre } 25 \text { e } 64 \\
\text { anos), apesar da baixa } \\
\text { cobertura do rastreamento } \\
\text { considerando a população } \\
\text { do município. Foram } \\
\text { poucas as amostras } \\
\text { consideradas insatisfatórias. } \\
\text { Em contrapartida, um } \\
\text { número considerável das } \\
\text { amostras não obteve } \\
\text { representatividade da } \\
\text { junção escamo-colunar, este } \\
\text { constitui um ponto crítico a } \\
\text { ser melhorado na eficácia } \\
\text { do exame, uma vez que, } \\
\text { verificou-se associação } \\
\text { significativa entre a } \\
\text { representação dos epitélios } \\
\text { e alterações anormais. }\end{array}$ \\
\hline $\begin{array}{l}\text { Leite, Nunes, } \\
\text { Oliveira, Barbosa e } \\
\text { Teles. (2019)/ } \\
\text { LILACS }\end{array}$ & $\begin{array}{c}\text { Montes Claros } \\
\text { (MG) }\end{array}$ & $\begin{array}{l}\text { Descrever a } \\
\text { percepção das } \\
\text { mulheres idosas } \\
\text { sobre o exame }\end{array}$ & $\begin{array}{l}\text { Estudo descritivo com } \\
\text { abordagem qualitativa } \\
\qquad(\mathrm{n}=12)\end{array}$ \\
\hline
\end{tabular}




\begin{tabular}{|c|c|c|c|}
\hline & & $\begin{array}{c}\text { preventivo do } \\
\text { câncer de colo de } \\
\text { útero (PCCU). }\end{array}$ & $\begin{array}{c}\text { A maioria das idosas } \\
\text { entrevistadas possui o } \\
\text { entendimento empírico } \\
\text { sobre o exame preventivo, } \\
\text { considerando-o importante, } \\
\text { todavia muitas delas } \\
\text { indagam sentimentos de } \\
\text { vergonha e medo ao se } \\
\text { submeterem ao exame. A } \\
\text { orientação quanto à } \\
\text { periodicidade da realização } \\
\text { do preventivo é feita pelos } \\
\text { profissionais de saúde, } \\
\text { porém muitas não o } \\
\text { realizam. }\end{array}$ \\
\hline $\begin{array}{l}\text { Moreira e Carvalho. } \\
\text { (2020)/LILACS }\end{array}$ & $\begin{array}{c}\text { João Pessoa } \\
\text { (PB) }\end{array}$ & $\begin{array}{c}\text { Conhecer a } \\
\text { tendência temporal } \\
\text { de realização da } \\
\text { citologia oncótica e } \\
\text { identificar fatores } \\
\text { associados à } \\
\text { realização do } \\
\text { exame em algum } \\
\text { momento da vida e } \\
\text { nos últimos três } \\
\text { anos. }\end{array}$ & $\begin{array}{l}\text { Estudo epidemiológico } \\
\text { (n=736) } \\
\text { Foi observada estabilidade } \\
\text { na realização da citologia } \\
\text { oncótica no período de } 2007 \\
\text { a } 2015 \text {. Entre as variáveis } \\
\text { analisadas, a faixa etária } \\
\text { esteve associada a maior } \\
\text { prevalência de realização do } \\
\text { exame nos dois desfechos } \\
\text { (em algum momento da } \\
\text { vida e nos últimos } 3 \text { anos); } \\
\text { o estado civil casado/união } \\
\text { estável manteve-se } \\
\text { associado à maior } \\
\text { prevalência de realização do } \\
\text { exame em algum momento } \\
\text { da vida; e a escolaridade de } \\
9 \text { a } 11 \text { anos de estudo } \\
\text { apresentou uma associação } \\
\text { negativa com a realização } \\
\text { do exame nos últimos três } \\
\text { anos. }\end{array}$ \\
\hline $\begin{array}{c}\text { Melo, Linhares, } \\
\text { Silva, Pontes, Santos } \\
\text { e Oliveira. } \\
\text { (2019)/SciELO }\end{array}$ & Recife (PE) & $\begin{array}{c}\text { Avaliar o } \\
\text { conhecimento, } \\
\text { atitude e práticas de } \\
\text { mulheres sobre o } \\
\text { exame preventivo } \\
\text { do câncer cérvico- } \\
\text { uterino e investigar } \\
\text { sua associação com } \\
\text { as variáveis } \\
\text { sociodemográficas. }\end{array}$ & $\begin{array}{c}\text { Estudo transversal analítico } \\
\text { quantitativo } \\
(\mathrm{n}=500) \\
\text { A prevalência de } \\
\text { conhecimento, atitude e } \\
\text { prática adequados foi de } \\
35,2 \%, 98 \% \text { e } 70,6 \%, \\
\text { respectivamente. } \mathrm{O}\end{array}$ \\
\hline
\end{tabular}




\begin{tabular}{|c|c|c|c|}
\hline & & & $\begin{array}{l}\text { conhecimento adequado foi } \\
\text { associado a não ter filhos, } \\
\text { ter renda familiar de dois } \\
\text { salários mínimos e religião } \\
\text { espírita/afro-brasileira. }\end{array}$ \\
\hline $\begin{array}{l}\text { Paula, Ferreira, } \\
\text { Marin, Meneguin e } \\
\text { Ferreira. } \\
\text { (2019)/LILACS }\end{array}$ & $\begin{array}{c}\text { Município do } \\
\text { interior (SP) }\end{array}$ & $\begin{array}{l}\text { Apreender os } \\
\text { saberes de } \\
\text { mulheres sobre a } \\
\text { prevenção do } \\
\text { câncer de colo } \\
\text { uterino por meio do } \\
\text { exame de } \\
\text { Papanicolau. }\end{array}$ & $\begin{array}{c}\text { Estudo descritivo } \\
\text { qualitativo } \\
(\mathrm{n}=20) \\
\text { As mulheres referiram } \\
\text { sentimentos de desconforto, } \\
\text { incomodo, vergonha e } \\
\text { medo. Quanto ao } \\
\text { conhecimento, } \\
\text { apresentaram-se deficientes, } \\
\text { em relação a finalidade do } \\
\text { exame e a própria doença. }\end{array}$ \\
\hline $\begin{array}{l}\text { Albuquerque, } \\
\text { Miranda, Leite e } \\
\text { Leite (2016) } \\
\text { /BDENF. }\end{array}$ & $\begin{array}{c}\text { Cuiá/João } \\
\text { Pessoa (PB) }\end{array}$ & $\begin{array}{c}\text { Analisar o } \\
\text { conhecimento de } \\
\text { mulheres acerca do } \\
\text { exame preventivo } \\
\text { do câncer de colo } \\
\text { do útero (CCU), } \\
\text { bem como verificar } \\
\text { a prática do exame. }\end{array}$ & $\begin{array}{l}\text { Exploratório descritivo } \\
\text { (n=30) } \\
\text { O conhecimento das } \\
\text { mulheres envolvidas na } \\
\text { pesquisa possuía sobre o } \\
\text { exame preventivo do CCU } \\
\text { era superficial. Todas } \\
\text { consideram relevante a } \\
\text { realização do mesmo e } \\
\text { apresentaram } \\
\text { esclarecimento acerca dos } \\
\text { cuidados preventivos do } \\
\text { exame, além de 86,67\% } \\
\text { declaram ter recebido } \\
\text { alguma informação do } \\
\text { exame. }\end{array}$ \\
\hline $\begin{array}{l}\text { Neves et al. } \\
\text { (2016)/BDENF }\end{array}$ & $\begin{array}{l}\text { Município do } \\
\text { interior do } \\
\text { estado do } \\
\text { Ceará }\end{array}$ & $\begin{array}{c}\text { Descrever a } \\
\text { percepção de } \\
\text { usuárias acerca do } \\
\text { exame de detecção } \\
\text { precoce do câncer } \\
\text { de colo uterino. }\end{array}$ & $\begin{array}{c}\text { Pesquisa descritiva } \\
\text { desenvolvida em cinco } \\
\text { Unidades de Saúde. } \\
\text { As entrevistadas percebem } \\
\text { o exame como importante e } \\
\text { que eventuais motivos para } \\
\text { a recusa podem ser evitados } \\
\text { por meio do fortalecimento } \\
\text { de vínculos no serviço. }\end{array}$ \\
\hline $\begin{array}{l}\text { Oliveira et al. } \\
\text { (2016)/BDENF }\end{array}$ & $\begin{array}{c}\text { João Pessoa } \\
\text { (PB) }\end{array}$ & $\begin{array}{c}\text { Avaliar o perfil } \\
\text { sociodemográfico e } \\
\text { a adesão das } \\
\text { mulheres ao exame }\end{array}$ & $\begin{array}{l}\text { Exploratório descritivo } \\
\qquad(\mathrm{n}=384)\end{array}$ \\
\hline
\end{tabular}




\begin{tabular}{|c|c|c|c|}
\hline & & $\begin{array}{c}\text { citológico do colo } \\
\text { uterino na Atenção } \\
\text { Básica. }\end{array}$ & $\begin{array}{l}\text { 96,4\% das entrevistadas } \\
\text { referiram ter realizado o } \\
\text { exame citológico ao menos } \\
\text { uma vez e possuía } \\
\text { periodicidade anual. } \\
\text { Vergonha, ansiedade, prazo } \\
\text { para recebimento do } \\
\text { resultado do exame, foram } \\
\text { os principais fatores que } \\
\text { dificultam a realização do } \\
\text { citológico. }\end{array}$ \\
\hline $\begin{array}{l}\text { Rodrigues et al. } \\
\text { (2016)/SciELO. }\end{array}$ & $\begin{array}{c}\text { Região oeste } \\
\text { de Minas } \\
\text { Gerais }\end{array}$ & $\begin{array}{c}\text { Analisar os } \\
\text { indicadores do } \\
\text { rastreamento do } \\
\text { câncer do colo do } \\
\text { útero na Região } \\
\text { Ampliada Oeste de } \\
\text { Minas Gerais, } \\
\text { no período de } 2007 \\
\text { a } 2013 .\end{array}$ & $\begin{array}{c}\text { Estudo epidemiológico } \\
\text { descritivo, com análise } \\
\text { ecológica. } \\
\text { A cobertura de exames } \\
\text { variou de } 61,0 \% \text { a } 74,0 \% \text {. A } \\
\text { maioria tinha entre } 35 \text { e } 54 \\
\text { anos. Foram consideradas } \\
\text { insatisfatórias } 0,86 \% \text { das } \\
\text { lâminas analisadas. O } \\
\text { epitélio escamoso foi o mais } \\
\text { evidenciado (99,1\%) nas } \\
\text { amostras. Observou-se a } \\
\text { presença de inflamação em } \\
41,9 \% \text { dos exames. } \\
\text { Prevalência de células } \\
\text { atípicas escamosas de } \\
\text { significado indeterminado, } \\
\text { possivelmente não } \\
\text { neoplásico, e as lesões } \\
\text { intraepiteliais de baixo grau. } \\
\text { Algumas variáveis } \\
\text { apresentaram incompletude. }\end{array}$ \\
\hline $\begin{array}{l}\text { Aguilar e Soares } \\
\text { (2015)/SciELO. }\end{array}$ & $\begin{array}{l}\text { Vitória da } \\
\text { Conquista } \\
\text { (BA) }\end{array}$ & $\begin{array}{c}\text { Conhecer as } \\
\text { barreiras que levam } \\
\text { mulheres em idade } \\
\text { fértil da cidade de } \\
\text { Vitória da } \\
\text { Conquista-BA a } \\
\text { não realizarem o } \\
\text { exame Papanicolau, } \\
\text { na perspectiva das } \\
\text { próprias mulheres e } \\
\text { dos profissionais de } \\
\text { saúde. }\end{array}$ & $\begin{array}{c}\text { Estudo descritivo, } \\
\text { exploratório qualitativo } \\
\text { (n=26) } \\
\text { Foi aplicada análise } \\
\text { categorial temática, que } \\
\text { revelou as seguintes } \\
\text { barreiras para não } \\
\text { realização do exame: } \\
\text { conhecimento insuficiente, } \\
\text { sentimentos negativos, falta } \\
\text { de atitude, aspectos } \\
\text { vinculados aos serviços de } \\
\text { saúde e inserção da mulher } \\
\text { no mercado de trabalho. }\end{array}$ \\
\hline
\end{tabular}




\begin{tabular}{|c|c|c|c|}
\hline $\begin{array}{l}\text { Santos, Holanda, } \\
\text { Silva, Santos e Silva } \\
\text { (2015)/ LILACS. }\end{array}$ & Maceió (AL) & $\begin{array}{c}\text { Analisar o } \\
\text { conhecimento das } \\
\text { mulheres em } \\
\text { relação à prevenção } \\
\text { do câncer de colo } \\
\text { do útero e os } \\
\text { fatores } \\
\text { dificultadores } \\
\text { acerca da } \\
\text { realização da } \\
\text { prática do exame } \\
\text { preventivo. }\end{array}$ & $\begin{array}{c}\text { Estudo observacional, de } \\
\text { corte transversal e } \\
\text { descritivo. } \\
\text { (n=110) } \\
\text { Dados referentes ao } \\
\text { significado do câncer } \\
\text { uterino mostraram que } 65 \\
(59,1 \%) \text { desconheciam seu } \\
\text { significado, } 69 \text { (62,7\%) } \\
\text { sabiam como preveni-lo, } \\
104 \text { (94,5\%) já realizaram o } \\
\text { Papanicolau, } 59 \text { (53,6\%) } \\
\text { realizaram o Papanicolau há } \\
1 \text { ano, } 62 \text { (56,4\%) realizam } \\
\text { o exame preventivo } \\
\text { anualmente e } 88 \text { ( } 80 \%) \\
\text { sabiam a importância dessa } \\
\text { realização. Quanto aos } \\
\text { fatores encontrados } \\
\text { referentes à dificuldade na } \\
\text { realização do exame } \\
\text { preventivo, } 49 \text { (44,5\%) } \\
\text { relataram ser a vergonha o } \\
\text { fator mais impactante. }\end{array}$ \\
\hline $\begin{array}{l}\text { Silva, Teixeira, } \\
\text { Ferrari, Cestari e } \\
\text { Cardelli } \\
(2015) / \text { LILACS. }\end{array}$ & Londrina (PR) & $\begin{array}{l}\text { Identificar motivos } \\
\text { para baixa adesão } \\
\text { ao exame de } \\
\text { Papanicolau entre } \\
\text { mulheres atendidas } \\
\text { na atenção primária } \\
\text { de saúde. }\end{array}$ & $\begin{array}{c}\text { Estudo transversal } \\
\text { (n=169) } \\
\text { Das } 169 \text { mulheres, 67\% } \\
\text { estavam em idade } \\
\text { reprodutiva e } 73,9 \% \\
\text { cursaram o ensino } \\
\text { fundamental. O não } \\
\text { comparecimento para o } \\
\text { exame previamente } \\
\text { agendado foi devido, } \\
\text { principalmente, às crenças e } \\
\text { atitudes (36,1\%) e à } \\
\text { organização do serviço } \\
\text { (25,4\%). Os sentimentos } \\
\text { referidos pelas mulheres } \\
\text { durante o Papanicolau } \\
\text { foram vergonha (55,6\%), } \\
\text { desconforto }(32,5 \%) \text { e dor } \\
\text { (20,7\%). }\end{array}$ \\
\hline $\begin{array}{l}\text { Amorim e Barros } \\
\text { (2014)/SciELO }\end{array}$ & $\begin{array}{l}\text { Campinas } \\
\text { (SP) }\end{array}$ & $\begin{array}{c}\text { Analisar a } \\
\text { desigualdade social } \\
\text { na prevalência da }\end{array}$ & $\begin{array}{l}\text { Estudo transversal de base } \\
\text { populacional. }\end{array}$ \\
\hline
\end{tabular}




\begin{tabular}{|c|c|c|c|}
\hline & & $\begin{array}{c}\text { realização do } \\
\text { exame de } \\
\text { Papanicolau nos } \\
\text { últimos três anos } \\
\text { segundo filiação a } \\
\text { planos privados de } \\
\text { saúde e, também, } \\
\text { em relação a outras } \\
\text { variáveis } \\
\text { socioeconômicas, } \\
\text { demográficas e de } \\
\text { comportamentos } \\
\text { relacionados à } \\
\text { saúde em mulheres } \\
\text { de } 20 \text { a } 59 \text { anos, } \\
\text { residentes no } \\
\text { município } \\
\text { Campinas (SP). }\end{array}$ & $\begin{array}{c}\text { (n=507) } \\
\text { Apesar das significativas } \\
\text { diferenças socioeconômicas } \\
\text { existentes entre as mulheres } \\
\text { filiadas e as não filiadas a } \\
\text { planos privados de saúde, } \\
\text { não foram observadas } \\
\text { diferenças na realização do } \\
\text { Papanicolau entre os dois } \\
\text { grupos, bem como em } \\
\text { relação a todas as outras } \\
\text { variáveis socioeconômicas } \\
\text { de saúde analisadas. } \\
\text { Somente a situação } \\
\text { conjugal revelou-se } \\
\text { associada à realização do } \\
\text { exame. O SUS foi } \\
\text { responsável pela cobertura } \\
\text { de 55,7\% dos exames } \\
\text { realizados. } \\
\end{array}$ \\
\hline $\begin{array}{l}\text { Nascimento e Araújo } \\
\text { (2014)/BDENF. }\end{array}$ & $\begin{array}{l}\text { Divinópolis } \\
\text { (MG) }\end{array}$ & $\begin{array}{l}\text { Conhecer as } \\
\text { motivações das } \\
\text { mulheres que não } \\
\text { realizam de forma } \\
\text { periódica o exame. }\end{array}$ & $\begin{array}{c}\text { Estudo qualitativo. } \\
\text { Os resultados mostram que } \\
\text { as motivações estão } \\
\text { relacionadas a sentimentos, } \\
\text { pouca informação, } \\
\text { sobrecarga do cotidiano, } \\
\text { dificuldades na relação } \\
\text { profissional-usuária e } \\
\text { barreiras institucionais. }\end{array}$ \\
\hline $\begin{array}{c}\text { Silva et al } \\
(2014) / \text { SciELO. }\end{array}$ & Maranhão & $\begin{array}{l}\text { Analisar o } \\
\text { rastreamento do } \\
\text { câncer do colo do } \\
\text { útero no Maranhão, } \\
\text { através dos dados } \\
\text { do Sistema de } \\
\text { Informação do } \\
\text { Câncer do Colo do } \\
\text { Útero (Siscolo). }\end{array}$ & $\begin{array}{c}\text { Estudo descritivo } \\
\text { retrospectivo, } \\
(\mathrm{n}=139.505)\end{array}$ \\
\hline
\end{tabular}




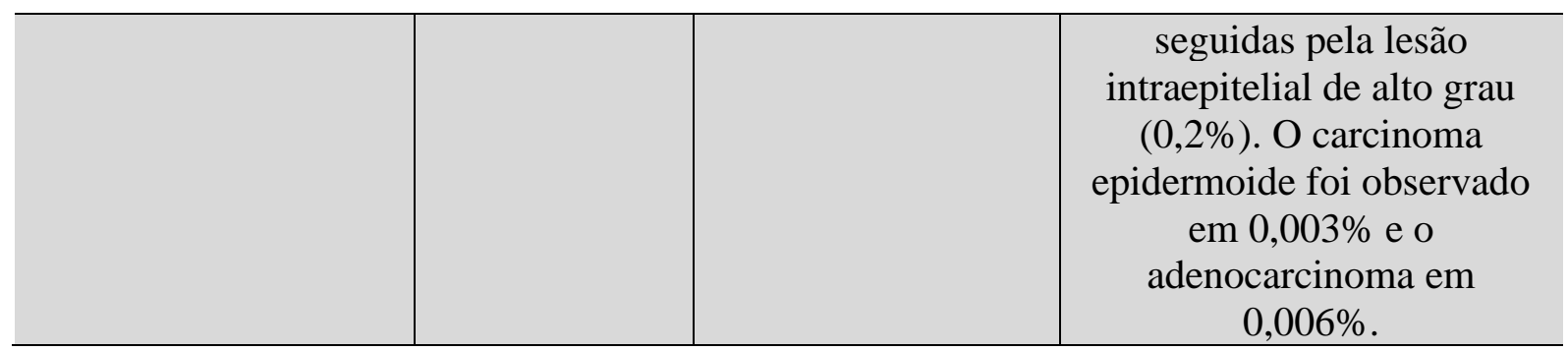

Fonte: Elaborada pelos autores (2020).

Após a análise dos artigos selecionados, os aspectos levantados foram agrupados nas seguintes categorias:

\subsection{Principais fatores de adesão e não adesão à realização do exame Papanicolau}

A prática do exercício do exame preventivo do câncer do colo do útero é imprescindível devido ao aumento da prevalência e mortalidade que esta patologia apresenta. Anualmente, são diagnosticados quase meio milhão de casos novos no mundo (Albuquerque et al., 2016).

Oliveira et al. (2016) relatam em seus estudos, que o recebimento de informações sobre o exame Papanicolau antes da sua realização foi o fator mais importante para adesão ao mesmo. Em seguida, destacaram os trabalhos realizados pelos profissionais de saúde em espaços de diálogo dentro e fora das unidades de saúde, seguido da prevenção do câncer do colo uterino, e um bom atendimento do profissional de saúde, sendo o último de extrema valia para a adesão das mulheres à realização da prevenção da doença.

Nos estudos de Neves et al. (2016), as mulheres demostraram estar cientes da importância da realização do exame e que a informação sobre o câncer de colo do útero representa relevante critério que contribui para a adesão ao exame. Quando o exame é realizado sem esclarecimento da sua significação e da sua importância, ele pode causar sentimentos negativos na mulher, comprometendo o diagnóstico precoce e interferindo na procura e adesão ao exame.

Para Albuquerque et al. (2016), a adesão das mulheres ao exame Papanicolau, está profundamente relacionada ao grau de conhecimento delas. A maioria das entrevistadas relatou saber a finalidade do exame preventivo do câncer do colo do útero, mesmo sendo um conhecimento genérico. Então, eles destacam que existe a necessidade de esclarecimento em relação à importância da realização do exame, abordando a prevenção.

Corroborando com esses dados, Nascimento e Araújo (2014) destacam que a educação eleva o grau de alerta para a importância da realização de exames preventivos e, em 
consequência pode melhorar a forma como o indivíduo compreende a informação sobre as avaliações, comunicação com ao profissional de saúde e a interpretação dos resultados. Assim, ressaltaram a ocorrência da incompreensão das orientações recebidas e o desconhecimento do profissional médico sobre a necessidade e indicação do exame de prevenção.

Moreira e Carvalho (2020) também abordaram em seus estudos a relação entre anos de escolaridade e a realização de exames preventivos, porém, mostrou resultados que foram discordantes aos de Albuquerque et al. (2016) e de Nascimento e Araújo (2014). Das mulheres com 0-8 anos, 84,2\% realizaram o exame em algum momento da vida, das com 9 a 11 anos, $76,3 \%$ e com 12 anos ou mais, 78,8\%. Quanto à realização do exame nos últimos 3 anos, de 08 anos foi de 75,6\%, de 9 a 11 anos foi de 68,4\% e com 12 anos ou mais, 75\%. Logo, ressaltase que os anos de escolaridade podem interferir no conhecimento sobre a importância da realização do exame, mas não é determinante nesse aspecto, o que amplia a discussão para outros aspectos que possam estar envolvidos para o grau de conhecimento sobre o exame.

Silva et al. (2015) descrevem que os sentimentos de vergonha e constrangimento é um dos principais motivos da não adesão à consulta, seguidos pela falta de conhecimento do câncer do colo do útero, da técnica e da importância do exame preventivo independente da faixa etária. Como fator principal a não adesão para a realização do exame, os autores citam a vergonha, sendo que este sentimento pode causar até a descontinuidade da assistência à mulher.

Dados semelhantes são encontrados em outros estudos. Sentimentos como a vergonha, ansiedade, medo e insegurança são presentes entre as mulheres durante os questionamentos a respeito dos aspectos que dificultam na realização do exame Papanicolau. Como principal sentimento desafiador para a realização do exame, os autores destacam a vergonha, fator que dificulta o acesso das mulheres ao exame, embora existam mulheres que referiam não ter vergonha de realizar o mesmo (Oliveira et al., 2016; Paula et al., 2019).

Em estudo realizado por Leite et al. (2020), analisaram-se entrevistas de doze idosas na faixa etária de 65 a 93 anos. As participantes eram na maioria viúvas, analfabetas e aposentadas. Apesar do conhecimento sobre o exame e sua importância, sentimentos relatados por outros estudos como medo, ansiedade, nervosismo estavam presentes em seus discursos quanto à realização do exame. Porém, a grande maioria relatou também desconforto ao realiza-lo, daí a relevância de atentar às particularidades da fisiologia genital de idosas, caracterizada por secura e atrofia vaginal por diminuição hormonal após a menopausa.

Em relação a possíveis fatores de não adesão à realização do exame ginecológico, Neves et al. (2016), apontam o desconhecimento sobre a importância do exame, a vergonha, o medo em relação ao câncer e ao próprio exame, inatividade sexual, embaraço, dor, nível 
socioeconômico e questões cultural podem ameaçar a adesão ao exame, pois o mesmo é visto por muitas mulheres como um exame que invade a privacidade e a integralidade corporal da mulher.

Em sua pesquisa, Aguilar e Soares (2015), mostram que um dos principais fatores para a não realização do exame Papanicolau, foi o descuido com a própria saúde. Muitas mulheres, somente se dirigem à assistência a saúde quando já estão doentes, indicando que isso acontece devido ao maior enfoque dado ao tratamento e não à prevenção das patologias, sendo influenciado pelo modelo biomédico ainda predominante no Brasil.

Dentre outros fatores para a não adesão a realização do exame Papanicolau, podemos citar a falta de recursos materiais, conhecimento da rotina do serviço e informações e esclarecimentos durante a consulta ginecológica para o exame preventivo. Portanto, são inúmeras barreiras que prejudicam uma prevenção correta e dificultam a continuidade à assistência (Nascimento \& Araújo, 2014).

\subsection{Fatores de risco para o câncer do colo do útero}

O câncer do colo do útero é mais presente em mulheres de classes sociais mais baixas e menor nível de escolaridade, pois a aquisição reduzida à informação sobre cuidados de saúde pode estar relacionada ao baixo nível de escolaridade, fato que pode refletir diretamente na demanda dos exames preventivos, na melhor compreensão das informações sobre as patologias e na necessidade de atitudes favoráveis a detecção precoce das neoplasias. As mulheres com baixo grau de instrução têm maior risco de desenvolver câncer do colo uterino, com uma relação indireta entre o grau de instrução e o risco do diagnóstico avançado do tumor (Santos et al., 2015).

Em suas pesquisas, Rodrigues et al. (2016), descrevem alguns fatores que podem influenciar no aparecimento do câncer do colo do útero, dentre eles, a imunidade, o tabagismo, a vida sexual precoce, a multiplicidade de parceiros sexuais, multiparidade, o uso de contraceptivos orais, a contaminação pelo Vírus da Imunodeficiência Humana (HIV) e outras doenças sexualmente transmissíveis.

Amorim e Barros (2014) destacam que, além dos fatores de riscos citados acima, a infecção persistente por alguns tipos de Papiloma Vírus Humano (HPV), quando ocorre a sua interação com outros fatores, apresenta-se também como importante fator de risco para o surgimento de células percussoras do câncer do colo do útero. O HPV está presente em 99\% dos cânceres cervicais e em 70\% desses casos são detectados os tipos 16 e 18 . 
Research, Society and Development, v. 9, n. 11, e2339119006, 2020

(CC BY 4.0) | ISSN 2525-3409 | DOI: http://dx.doi.org/10.33448/rsd-v9i11.9006

Corroborando com esses dados, Silva et al. (2014) afirmam que além dos aspectos relacionados à infecção pelo HPV (subtipo, carga viral, infecção única ou múltipla), o tabagismo, a multiplicidade de parceiros sexuais, o uso de contraceptivos orais, múltiplos partos, baixa ingestão de vitaminas, iniciação sexual precoce e a coinfecção por agentes infecciosos como o Vírus da Imunodeficiência Humana (HIV) e Chlamydia trachomatis se constituem outros fatores de risco para o desenvolvimento do câncer do colo do útero.

Ceolin et al. (2020) expõe que há tipos de lesões celulares que podem predizer riscos maiores para o desenvolvimento do câncer de colo do útero, ressaltou que lesões intraepiteliais escamosas de alto grau (HSIL) e o carcinoma epidermoide invasor são exemplos desses riscos, por isso a necessidade de detectar precocemente e tratar adequadamente em atenção secundária. Ao realizar um estudo com 1583 exames citopatológicos de mulheres entre 14 e 84 anos, 0,5\% apresentaram HSIL e 0,06\% carcinoma epidermoide invasor. Identificou-se associação significativa entre a representação dos epitélios e alterações anormais. Em 32\% das amostras anormais, evidenciou-se epitélio escamoso associado ao glandular e 51\% de epitélio escamoso, glandular e metaplásico associados. Dessa forma, o estudo sugere que amostras com representação do epitélio escamoso associado ao glandular e/ou metaplásico tem mais chances de detectar lesões precursoras.

Com o progresso dos estudos dos fatores de risco ligados ao câncer de colo de útero, podem-se ampliar estratégias da prevenção primária e secundária, visando à proteção da população suscetível ao desenvolvimento desta patologia que corresponde às mulheres com baixas condições socioeconômicas, ao início precoce da atividade sexual, à multiplicidade de parceiros sexuais, ao tabagismo e como citados anteriormente, à infecção pelo HPV (Oliveira et al, 2016).

\subsection{Caracterização sociodemográfica}

Apesar da recomendação das sociedades médicas para a realização periódica do Papanicolau e de o mesmo ser ofertado em serviços de atenção básica, estudos nacionais e internacionais identificaram fatores demográficos, socioeconômicos e geográficos que limitam o acesso ao exame. Entre esses fatores, destacam idade, escolaridade, renda, situação conjugal, condições de saúde, filiação a planos de saúde e acesso aos serviços de saúde, mostrando a necessidade do desenvolvimento de estratégias para a captação das mulheres que não realizam o exame de forma adequada (Amorim \& Barros, 2014). 
Em suas pesquisas, Santos et al. (2015) relatam que em sua amostra a maioria das mulheres tinham entre 25 e 44 anos, quase a metade eram casadas, mais da metade não atingiram o ensino médio e tinham renda familiar de até 2 salários mínimos. Em relação ao nível de conhecimento sobre o câncer do colo do útero, foi considerado baixo, pois a procura pelos serviços de saúde ocorre em virtude de alguma queixa e não pela prevenção. Quanto ao exame preventivo, quase todas já tinham realizado pelo menos uma vez.

$\mathrm{Na}$ amostra de Silva et al. (2015), mais da metade das mulheres apresentavam-se em idade reprodutiva, entre 17 e 76 anos, algumas eram adolescentes, correspondendo menos de cinco por cento das entrevistadas. A maior parte vivia com companheiro e tinha três ou mais filhos. A raça autorreferida predominante foi a branca, quase a metade era do lar e mais da maioria estudaram apenas o ensino fundamental. O motivo para não comparecer a coleta do Papanicolau em maior evidência estava relacionado às crenças e atitudes em saúde, dentre esses determinantes, a vergonha foi o sentimento predominante, quanto à realização do exame.

Aguilar e Soares (2015) descrevem que das entrevistadas a maior parte tinha entre $18 \mathrm{e}$ 28 anos, metade possuía ensino fundamental incompleto, maioria tinham renda mensal igual ou inferior a um salário mínimo, mais de setenta por cento iniciaram a vida sexual com idade menor ou igual a 18 anos, grandes partes não utilizavam nenhum método contraceptivo e referiram possuir apenas um parceiro sexual no último ano. Apontaram como elementos limitantes para a realização do Papanicolau o conhecimento insuficiente, crenças e tatus das mulheres, falta de atitude das mesmas, sentimentos negativos, como medo e constrangimento, inserção no mercado de trabalho e aspectos relacionados aos serviços de saúde.

Melo et al. (2019) demonstrou em seu estudo epidemiológico que a maioria das mulheres analisadas que realizaram o exame Papanicolau tinham entre 40 e 59 anos, representando 53,6\% do total, a maioria tinha três ou mais filhos $(33,6 \%), 60,2 \%$ delas não trabalhavam, $46 \%$ tinham ensino fundamental completo ou incompleto, 43,1\% viviam com renda familiar de um salário mínimo, 50,8\% se declararam católicas e 66\% se autodeclaravam pardas ou mulatas. O estudo concluiu que a adequação do conhecimento foi significativamente influenciada pelo número de filhos, renda familiar e religião, e observou-se uma maior associação ao conhecimento adequado nas mulheres que não tiveram filhos, possuíam renda de dois salários mínimos $(41,1 \%)$ e seguiam outra religião, que não a católica ou a protestante $(52,4 \%)$. Os demais dados sociodemográficos não apresentaram significância na determinação do conhecimento. Quanto à atitude e à prática, nenhuma variável apresentou associação com a prevalência de adequação. 
Research, Society and Development, v. 9, n. 11, e2339119006, 2020

(CC BY 4.0) | ISSN 2525-3409 | DOI: http://dx.doi.org/10.33448/rsd-v9i11.9006

Em seus estudos Neves et al. (2016), afirmam que as mulheres entrevistadas se encontravam na faixa etária de 22 a 69 anos e a maioria eram casadas. Com relação ao nível de escolaridade, quarenta por cento tinham apenas o ensino fundamental, os outros quarenta o ensino médio, e vinte por cento da amostra eram analfabetas. Com relação ao significado do exame, todas sabiam que o intuito do exame é detecção precoce do câncer de colo de útero, mais da metade referiram realizar o exame anualmente e que não existe motivos para que se recusem fazer o exame, alegando a importância de cuidar de sua saúde.

Ceolin et al. (2020) descreveu um estudo retrorspectivo feito em uma cidade do Rio Grande do Sul, onde se analisaram 1583 exames citopatológicos. A idade das mulheres que realizaram os exames variou de 14 a 84 anos, sendo a média de 43,9 anos. 1271 deles foram realizados em mulheres entre 25 e 64 anos, faixa etária da qual a incidência do câncer de colo do útero é maior. A faixa etária de 45 a 54 anos foi a que mais buscou a realização do exame citopatológico, sendo um dado positivo, pois lesões malignas mais graves acometem principalmente mulheres na faixa etária entre 35 e 55 anos.

Neste outro trabalho, a amostra foi composta por mulheres que variaram a idade entre 16 e 65 anos. Quanto à realização do exame citológico, a maioria afirma ter feito, alguma vez durante toda a vida, e uma minoria responderam que nunca realizaram o exame. Uma grande parte declarara possuir alguma periodicidade na realização do exame preventivo, predominando a realização anual, as outras não possui uma periodicidade definida, ou seja, o exame como algo presente na sua rotina de cuidado com a saúde (Oliveira et al., 2016).

\section{Considerações Finais}

Os resultados deste estudo revelaram diversos fatores que influenciam a mulher a realizar o exame preventivo do câncer do colo do útero, e em número mais significativo os fatores que levam as mulheres a não aderir ao exame.

Dentre os que levam a aderir o exame, constatou-se o recebimento de informações antes da realização do exame, o trabalho realizado pelos profissionais de saúde em espaços de diálogo dentro e fora das unidades de saúde, prevenção do câncer do colo do útero, bom atendimento profissional, importância da realização do exame, informações sobre a patologia, grau de conhecimento das mulheres e educação. Já aqueles fatores que limitam fora constatado o sentimento de vergonha e constrangimento, crenças, a falta de conhecimento do câncer do colo do útero e da técnica, desconhecimento da importância do exame, ansiedade, medo, insegurança, inatividade sexual, nível socioeconômico, questões cultural, descuido com a 
própria saúde, falta de recursos materiais, conhecimento da rotina do serviço e informações e esclarecimento durante a consulta ginecológica para o exame preventivo.

Percebeu-se, que as há uma limitação da realização do exame citopatológico nas mulheres de baixa renda, com baixo nível de escolaridade, conhecimento insuficiente sobre o exame e patologia e principalmente as que possuem o sentimento de vergonha em relação à realização do exame. Portanto, que esse estudo possibilitou ampliar os conhecimentos com relação aos fatores de adesão e não adesão à realização do exame citopatológico com a análise de trabalhos que comprovam a eficácia do rastreamento e os benefícios do Teste de Papanicolau na detecção precoce do câncer do colo do útero. Por conseguinte, espera-se que os resultados aqui obtidos possibilitem a realização de novos estudos.

\section{Referências}

Aguilar, R. P., \& Soares, D. A. (2015). Barreiras à realização do exame Papanicolau: perspectivas de usuárias e profissionais da Estratégia de Saúde da Família da cidade de Vitória da Conquista-BA. Revista de Saúde Coletiva, 25(2), 359-379.

Albuquerque, V. R., Miranda, R. V., Leite, C. A., \& Leite, M. C. A. (2016). Exame preventivo do câncer de colo do útero: conhecimento de mulheres. Ver. Enferm. UFPE on line, 10(5), 4208-4218.

Amorim, V. M, S. L., \& Barros, M. B. A. (2014). Equidade no acesso ao exame de Papanicolau: estudo de base populacional no município de Campinas, São Paulo, Brasil. Revista Brasileira de Epidemiologia, 17(Suppl. 2), 136-149.

Botelho, L. L. R., Cunha, C. C. A., \& Macedo, M. (2011). O método de revisão integrativa nos estudos organizacionais. Revista Eletrônica Gestão e Sociedade, 5 (11), 121-136.

Brasil, Ministério da Saúde, Departamento de Informática do Sistema único de Saúde -

DATASUS. Estatísticas Vitais: Mortalidade por Neoplasia Maligna do Colo do Útero. Recuperado de: http://tabnet.datasus.gov.br/cgi/tabcgi.exe?sim/cnv/obt10uf.def 
Ceolin, R., Nasi, C., Coelho, D. F., Paz, A. A., \& Lacchini, A. J. B. (2020). Análise do rastreamento do câncer do colo do úterode um município do sul do Brasil. Rev Fun Care Online, $12,406-412$.

Instituto Nacional de Câncer José Alencar Gomes da Silva - INCA. (2019). Estimativa 2020: incidência de câncer no Brasil.

Leite, B. O., Nunes, C. R. O., Oliveira, V. V., Barbosa, R. A. A., \& Teles, M. A. B. (2019). A Percepção das Mulheres Idosas Sobre o Exame de Prevenção de Câncer do Colo de Útero. Rev Fund Care Online, 11(5), 1347-1352.

Melo, E. M. F., Linhares, F. M. P., Silva, T. M., Pontes, C. M., Santos, A. H. S., \& Oliveira, S. C. (2019). Câncer cervico-uterino: conhecimento, atitude e prática sobre o exame de prevenção. Rev Bras Enferm, 72(3), 30-6.

Moreira, A. P. L., \& Carvalho, A. T. (2020). Tendência de Realização da Citologia Oncótica e Fatores Associados em Mulheres de 25 a 64 anos. Revista Brasileira de Ciências da Saúde, 24(1), 17-28.

Nascimento, G. W. C., Pereira, C. C. A., Nascimento, D. I. C., Lourenço, G. C., \& Machado, C. J. (2015). Cobertura do exame citopatológico do colo do útero no Estado de Minas Gerais, Brasil, no período entre 2000-2010: um estudo a partir dos dados do Sistema de Informação do Câncer do Colo do Útero (SISCOLO). Caderno Saúde Coletiva, 23(03), 253-260.

Nascimento, R. G., \& Araújo, A. (2014). Falta de periodicidade na realização do exame citopatológico do colo uterino: motivações das mulheres. Revista Mineira de Enfermagem, $18(3), 557-564$.

Neves, K. T. Q., Oliveira, A. W. N., Galvão, T. R. A. F., Ferreira, I. T., Mangane, E. M., \& Souza, L. B.(2016). Percepção de usuárias acerca do exame de detecção precoce do câncer de colo uterino. Cogitare Enfermagem, Paraná, 21(4), 01-07. 
Oliveira, A. E. C., Deininger, L. S. C., Lima, I. M. B., Lima, D. C., Nascimento, J. A., \& Andrade, J. M. (2016). Adesão das mulheres ao exame citológico do colo uterino na atenção básica. Revista Enfermagem UFPE on line, 10(11), 4003-14.

Paula, T. C., Ferreira, M. L. S. M., Marin, M. J. S., Meneguin, S., \& Ferreira, A. S. S. B. S. (2019). Detecção precoce e prevenção do câncer de colo uterino: saberes e práticas educativas. Enferm. Foco, 10(2), 47-51.

Pompeo, D. A., Rossi, L. A., \& Galvão, C. M. (2009) Revisão integrativa: etapa inicial do processo de validação de diagnóstico de enfermagem. Escola Paulista de Enfermagem, 22(4), 434-438.

Ribeiro, J. C., \& Andrade, S. R. (2016). Vigilância em saúde e a cobertura de exame citopatológico do colo do útero: revisão integrativa. Texto e Contexto Enfermagem, 25(4), e5320015.

Rodrigues, J. F., Moreira, B. A., Alves, T. G. S., \& Guimarães, E. A. A. (2016). Rastreamento do câncer de colo do útero na região ampliada oeste de Minas Gerais, Brasil. Revista de Enfermagem do Centro Oeste Mineiro, 06(2), 2156-2168.

Santos, A. M. R., Holanda, J. B. L., Silva, J. M. O., Santos, A. A. P., \& Silva, E. M. (2015). Câncer de colo uterino: conhecimento e comportamento de mulheres para a prevenção. Revista Brasileira de Promoção de Saúde, 28(2), 153-159.

Silva, D. S. M., Silva, A. M. N., Brito, L. M. O., Gomes, S. R. L., Nascimento, M. D.S. B., \& Chein, M. B. C. (2014). Rastreamento do câncer do colo do útero no Estado do Maranhão, Brasil. Ciência \& Saúde Coletiva, 19(4), 1163-1170.

Silva, M. A. S., Teixeira, E. M. B., Ferrari, R. A. P., Cestari, E. W., \& Cardelli, A. A. M. (2015). Fatores relacionados a não adesão à realização do exame de Papanicolau. Revista da Rede de Enfermagem do Nordeste, 16(4), 532-539.

Souza, A. F., \& Costa, L. H. R. (2015). Conhecimento de mulheres sobre HPV e câncer de colo de útero após consulta de enfermagem. Revista Brasileira de Cancerologia, 61(4), 343-350. 
Souza, M. T., Silva, M. D., \& Carvalho, R. (2010). Revisão integrativa: o que é e como fazer. Einsten, 8(1), 102-106.

\section{Porcentagem de contribuição de cada autor no manuscrito}

Giovanna Stefanne Lópes Barbosa - 10\%

Antônio Tiago da Silva Souza - 7,5\%

Francisco Clezion Franca Vasconcelos Júnior - 7,5\%

Edmar José Fortes Júnior - 5\%

Daniel Mayco de Melo Oliveira - 5\%

Francisco Lukas Rodrigues Martins - 5\%

Francisco Ricardo Nascimento Freitas - 5\%

Hyan Crysthyan Apolinário Silveira - 5\%

Ivy Louise Barros - 5\%

Leonardo Vitor Nunes de Oliveira - 5\%

Mariana Veras Rocha Borges - 5\%

Nayanna de Almeida Nassif Rodrigues - 5\%

Rafaela Sartori Tonin $-5 \%$

Raimundo Graças Almeida Lima Neto - 5\%

Samuel Aragão Cansanção Bona Ibiapina - 5\%

Carlos Alberto Teixeira Costa - 5\%

Daniela França de Barros - 5\%

José Ivo dos Santos Pedrosa - 5\% 